\title{
Modelación matemática y algorítmica de los términos de la teoría del socioconstructivismo para un entorno educativo digital
}

\section{Mathematical and algorithmic modeling of the terms of the theory of socioconstructivism for a digital educational environment}

\author{
Igor Ivanov \\ Universidad Tecnológica Empresarial de Guayaquil. Guayaquil, Ecuador \\ iivanov@uteg.edu.ec \\ Marina Kosonogova \\ Universidad Estatal Tecnológica de Belgorod. Belgorod, Rusia \\ marinakosonogovasc@gmail.com \\ Jesennia Cárdenas Cobo \\ Universidad Estatal de Milagro. Milagro, Ecuador \\ jcardenasc@unemi.edu.ec
}

\begin{abstract}
Resumen
Este trabajo presta especial atención al concepto de NGDLE (Next Generation Digital Learning Environment (ingles), Entorno Educativo Digital de Próxima Generación) y proporciona una lista de componentes típicos que pueden integrarse en el entorno educativo según el modelo NGDLE. Una de las limitaciones de sistemas e-learning es la dificultad de observar y corregir el proceso del aprendizaje individualmente. Generalmente, la variación del contenido individual de un curso se basa en decisión binaria: si la prueba se ha aprobado o no. En estos sistemas no se tiene en cuenta las características particulares del alumnado, no obstante, la teoría pedagógica ha logrado suficientes resultados. Los autores consideran la posibilidad de sumergir la teoría del socioconstructivismo de Lev Vygotsky en un entorno digital, particularmente su enfoque sobre el cambio sucesivo de zonas de desarrollo individuales. Debido a que en la actualidad no existen los modelos cuantitativos de zonas de desarrollo, entonces, se plantea y resuelve la tarea de construir un modelo matemático de la zona de desarrollo próximo, basado en la teoría del rasgo latente y psicometría computacional. Para estimar cuantitativamente la anchura de la zona del desarrollo próximo, utilizamos la idea de su proporcionalidad al nivel de capacitación, que, a su vez, se considera como una variable latente. Para una descripción formal del nivel de capacitación, se propone una lista de indicadores medibles: indicadores de actividad educativa y el patrón de interacción entre el usuario y el entorno digital. El modelo construido de la zona de desarrollo se utiliza para la gestión adaptativa del aprendizaje electrónico, en el que presenta un algoritmo de control que implementa el aprendizaje del desarrollo. Se describe la técnica de evaluar la conveniencia de aplicar el modelo de la zona de desarrollo proximal. Para evaluar la viabilidad, se ha utilizado el material experimental: datos sobre los resultados de las actividades educativas de 1469 estudiantes en dos países: Rusia y Ecuador. Como resultado del estudio, se confirmó la hipótesis de que el nivel de capacitación alcanzado en el curso con los elementos de desarrollo cognitivo es superior a los resultados del aprendizaje electrónico, sin tener en cuenta la teoría del socioconstructivismo. Además, se consideran enfoques de la arquitectura del moderno entorno educativo digital.
\end{abstract}


Palabras clave: desarrollo cognitivo, e-learning, nivel de capacitación, teoría de rasgos latentes, zonas de desarrollo, indicadores educativos, trayectoria de aprendizaje.

\begin{abstract}
The focus of the paper is on NGDLE (Next Generation Digital Learning Environment) concept and it provides a list of typical components that can be integrated into the educational environment according to the NGDLE model. One of the limitations of e-learning systems is the difficulty to observe and correct the learning process individually. Generally, variation of individual course content is based on binary decision: whether the test has been passed or not. The particular characteristics of the students are not taken into account by the systems, although pedagogical theory achieved sufficient results. The authors consider the possibility of immersing Lev Vygotsky's theory of socio-constructivism into a digital environment, particularly its focus on the continuous change of personal development zones. Since currently do not exist quantitative models of developmental zones, the task is posed and solved to construct a mathematical model of the proximal developmental zone, based on the item response theory and computational psychometrics. To quantitatively estimate the width of the zone of proximal development, we use the idea of its proportionality to the knowledge level, which, in turn, is considered as a latent variable. For a formal description of the knowledge level, we propose a list of measurable indicators: indicators of educational activity and the pattern of interaction between the user and the digital environment. The constructed model of the development zone is used for the adaptive management of e-learning, in which it presents a control algorithm that implements cognitive development. The technique of assessing the suitability of applying the model of the proximal development zone is described. To assess feasibility, the experimental material has been used: data on the results of educational activities of 1469 students in two countries: Russia and Ecuador. As a result of the study, the hypothesis was confirmed that the level of training achieved in the course with the elements of cognitive development is higher than the results of e-learning, without taking into account the theory of socio-constructivism. In addition, approaches to the architecture of the modern digital educational environment are considered.
\end{abstract}

Keywords: cognitive development, eLearning, knowledge level, item response theory, psychometrics, educational indicators, learning trajectory.

\title{
1. Introducción
}

En la etapa actual del desarrollo de la esfera de e-learning para la organización de aprendizaje se utilizan las soluciones de LMS (Learning Management System (ingles), Sistema de Gestión del Aprendizaje), a saber, eFront, Moodle, iSpring Learn, Talento LMS, Unicornio LMS, así mismo las plataformas de MOOC (Massive Open Online Courses (ingles), Cursos Masivos Abiertos en Línea) como por ejemplos Coursera, Udacity, Udemy, MIT OpenCourseWare, Stepik. Dichos tipos de software se posicionan como modelos típicos de un entorno educativo digital. A su vez, el fenómeno de la "economía digital", que implica el desarrollo de cualquier industria en forma de un ecosistema abierto basado en tecnologías de información, ha creado los requisitos previos para un nuevo modelo del entorno educativo NGDLE.

El concepto del sistema NGDLE implica combinar muchos servicios educativos y plataformas individuales en un único ecosistema para la plena implementación de la idea

Modelación matemática y algorítmica de los términos de la teoría del socioconstructivismo para un entorno educativo digital. Ivanov, I., Kosonogova, M., Cárdenas, J. 
de aprendizaje electrónico innovador. Los componentes típicos que se pueden integrar en el entorno educativo utilizando el modelo NGDLE se muestran en la Tabla 1 (Birjalia, Beni-Hssanea \& Erritalib 2018, Rodrigues, Isotani \& Zárate 2018, Tsatsou, Vretos \& Daras 2019).

Tabla 1. Componentes del entorno educativo del paradigma NGDLE

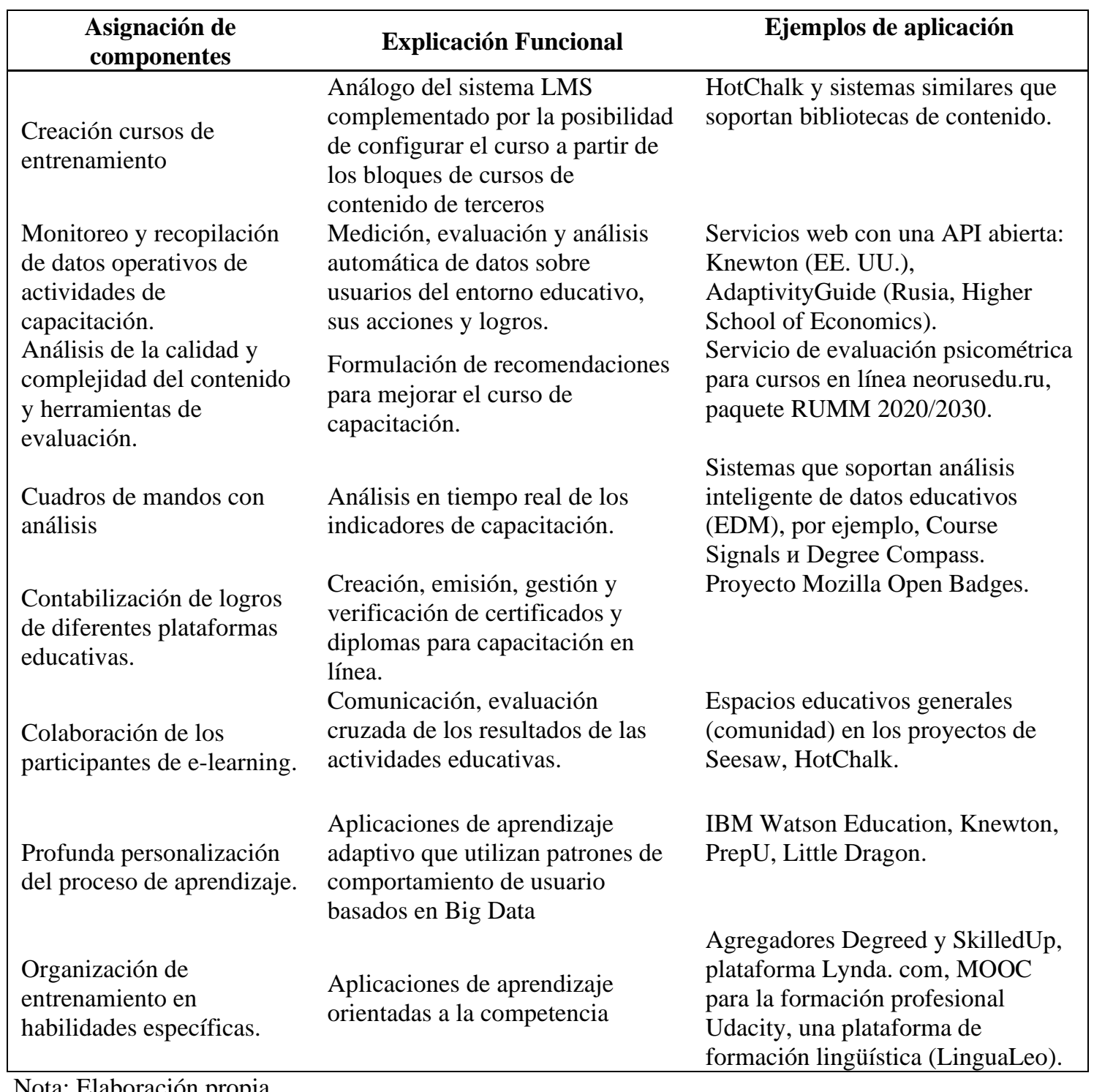

Como puede verse en la lista de componentes típicos, el entorno educativo digital de nueva generación utiliza un enfoque activo para el aprendizaje, en el que el enfoque se centra en el resultado y la participación en el proceso educativo. El rol de las tecnologías de la información ya no se limita a la automatización de la entrega unidireccional de los contenidos educativos (Lazo, Osuna-Acedo \& Quintana 2019). En estas condiciones, el desarrollo de bases teóricas y metodológicas para la formación de las acciones de control en los medios educativos digitales, es una nueva generación de soluciones que depende de los siguientes problemas principales:

1. Modificación de la experiencia de aprendizaje electrónico, basada en la transferencia de teorías científicas de aprendizaje al entorno educativo digital, formando modelos pedagógicos cuantitativos de aprendizaje electrónico.

Modelación matemática y algorítmica de los términos de la teoría del socioconstructivismo para un entorno educativo digital. Ivanov, I., Kosonogova, M., Cárdenas, J. 
2. El desarrollo y estudio de modelos, métodos y herramientas, para la evaluación a fondo de los conocimientos, las habilidades y las competencias de los educandos, sobre la base de pruebas de resultados del aprendizaje y de la interacción con el entorno de aprendizaje.

\section{Inmersión de las teorías del aprendizaje en un entorno digital}

Desde mediados del siglo XX, el desarrollo de los principios del aprendizaje automatizado ha sido influenciado por muchas tecnologías pedagógicas y teorías del aprendizaje (Bosco 2013, Ferreyra \& Pedrazzi 2007, Pozo, Scheuer, Pérez, Mateus \& Martín 2006, Vázquez \& Camacho 2008). Entre ellas: instrucción programada, aprendizaje basado en problemas, la teoría de la formación gradual de las acciones mentales, la teoría de los sistemas pedagógicos, la psicología cognitiva, desarrollo del aprendizaje, etc. Si se selecciona la base pedagógica para la implementación completa del aprendizaje adaptativo y personalizado, entonces la posibilidad de inmersión de la teoría del desarrollo del aprendizaje en un entorno digital debe ser considerada en primer lugar. Esto se justifica por el hecho de que, la teoría tiene en cuenta la relación entre el aprendizaje y el desarrollo individual. Entonces, el uso de la teoría del desarrollo del aprendizaje en formato digital, permitirá introducir un modelo de toma de decisiones promoviendo la dinámica de estructuración de la trayectoria educativa.

\subsection{Panorama general de la teoría del desarrollo del aprendizaje}

En la ciencia pedagógica, el desarrollo del aprendizaje se conoce bajo varios términos: teoría de zonas de desarrollo, constructivismo social, andamiaje.

El enfoque constructivista de la educación consiste en el hecho de que el conocimiento no puede ser transferido de forma prefabricada, por lo que el alumnado construye su propia comprensión del mundo, interpreta y estructura la información sobre los objetos de la cognición por sí mismo. Al mismo tiempo, el rol del maestro es crear el entorno y las condiciones educativas apropiadas para tal proceso. Es fundamental considerar que la percepción de las situaciones de aprendizaje está influida por muchos factores a conocer, tales como la edad, la personalidad, las características psicológicas del alumnado y el nivel de desarrollo de sus funciones cognitivas. Se debe señalar que los modernos instrumentos digitales de aprendizaje, tienen un importante potencial para la consideración objetiva de estos factores en el proceso de construcción de trayectorias educativas.

El constructivismo en la educación se ha desarrollado en dos direcciones. El constructivismo cognitivo introduce el principio de seguimiento del alumnado, según el cual es necesario organizar el entorno educativo de tal manera que le induzca a actuar de nuevo. Se cree que la verdadera naturaleza del aprendizaje que promueve el desarrollo se revela en el constructivismo social, que representa el aprendizaje como una colaboración mutuamente activa entre el profesor y el estudiante (Bodrova \& Leong 2007). La base del constructivismo social en la psicología y la pedagogía occidentales es la teoría históricocultural de Lev Vygotsky, que combina varias leyes de desarrollo y sus implicaciones pedagógicas. Según Vygotsky, el desarrollo de herramientas de aprendizaje se basa en la interconexión de la ley de sociogénesis (interacción social) y la ley de mediación (transformación del comportamiento), es decir, la transición de las formas naturales de comportamiento a las culturales (Zuckerman 2014).

El significado del desarrollo del aprendizaje es cambiar gradualmente de zonas de desarrollo como resultado de un material educativo de dificultad creciente (Vygotsky 2008). El cambio de las zonas de desarrollo es el siguiente: el material de capacitación se encuentra inicialmente en su zona de desarrollo posterior desde el punto de vista del

Modelación matemática y algorítmica de los términos de la teoría del socioconstructivismo para un entorno educativo digital. Ivanov, I., Kosonogova, M., Cárdenas, J.

Página 4 de 20 
autoestudio exitoso por parte de los estudiantes, la zona se caracteriza por el hecho de que el nivel de dificultad del material y el nivel del desarrollo de las habilidades del sujeto, necesarias para resolver el problema, no son comparables. En tales condiciones, el éxito de la capacitación no se puede lograr incluso con la participación de varios tipos de asistencia. En el nivel de desarrollo actual, los materiales con un nivel de dificultad que no exceda un umbral $\delta$ se pueden dominar independientemente. Desde la zona de desarrollo actual se abre la zona de desarrollo próximo, habiendo dominado con la ayuda de varios tipos de asistencia, el estudiante la "convierte" en una zona de desarrollo actual, etc.

La dificultad de usar la teoría del desarrollo del aprendizaje es que el constructo "zona de desarrollo" no es directamente evaluable y medible. Los resúmenes y estudios (Hakkarainen \& Korepanova 2009) sobre el tema de la educación para el desarrollo describen principalmente diversos enfoques para el diagnóstico psicológico y pedagógico de la zona de desarrollo próximo. No proporcionan modelos matemáticos rigurosos para describir las características de las zonas de desarrollo, lo cual es crítico para generar acciones de control en el proceso de e-learning. El uso de la teoría de zonas de desarrollo para la organización del aprendizaje automatizado se considera en un número relativamente pequeño de estudios (Lin, Tseng, Weng \& Su 2009, Tzuriel 2000). Al mismo tiempo, solo hay un criterio específico para la implementación del e-learning en la zona de desarrollo próximo: si el sujeto resuelve los problemas de dificultad $\boldsymbol{P}$ en el nivel de referencia, entonces él o ella resuelve problemas un $20 \%$ más difíciles que $\boldsymbol{P}$ con un $80 \%$ de confiabilidad. Sin embargo, el uso de un ancho fijo de la zona de desarrollo y los niveles de referencia de asimilación del material es controvertido en términos de aprendizaje para el desarrollo.

En general, la transformación de la teoría tradicional del aprendizaje en un análogo digital se puede describir mediante el esquema que se muestra en la Figura 1. En primer lugar, es necesario justificar la posibilidad fundamental de transferir una determinada teoría del aprendizaje al entorno digital, es decir, seleccionar las herramientas y los componentes del entorno educativo moderno adecuados para la implementación de las disposiciones clave de esta teoría.

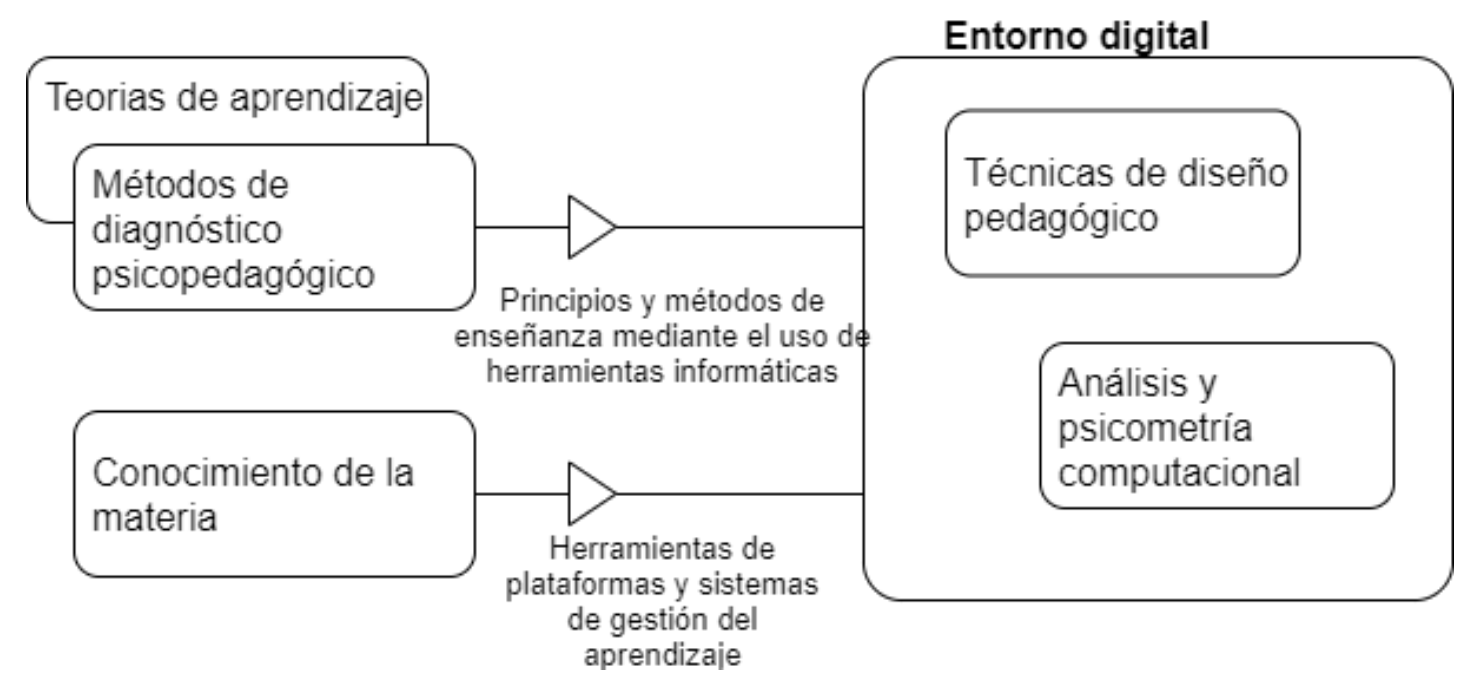

Figura 1. Esquema de inmersión de la teoría del aprendizaje en el entorno digital Nota: Elaboración propia.

Modelación matemática y algorítmica de los términos de la teoría del socioconstructivismo para un entorno educativo digital. Ivanov, I., Kosonogova, M., Cárdenas, J. 
Al implementar el esquema de inmersión de la teoría del aprendizaje en un entorno digital, el diseño pedagógico proporciona (Lin, Tseng, Weng, \& Su 2009):

- análisis de las necesidades del público objetivo, sus competencias y los resultados de aprendizaje esperados;

- la elección de medios y métodos de trabajo educativo;

- desarrollo de pruebas y tareas, creación de cursos utilizando herramientas apropiadas;

- cargar un curso en el sistema de gestión del aprendizaje;

- desarrollo de métodos para evaluar los resultados y la eficacia de los materiales;

- desarrollo de soluciones para la mejora del contenido educativo.

Las técnicas de psicometría computacional en el problema de sumergir la teoría del aprendizaje en un entorno digital se utilizan para:

- identificación e investigación de factores que aseguran el éxito del e-learning;

- análisis de la calidad de los programas y el contenido basado en datos que describen el comportamiento del alumnado en el e-learning;

- optimización de la ruta de aprendizaje basadas en datos.

El entorno digital permite organizar la gestión de las trayectorias educativas con base a varios tipos de analítica educativa: descriptiva, predictiva y prescriptiva (Rodrigues, Isotani \& Zárate 2018). El análisis descriptivo se utiliza para describir con precisión la situación actual de aprendizaje. La tarea principal es recopilar, estructurar y visualizar grandes conjuntos de datos que surgen de la interacción de los usuarios con el entorno educativo digital. Por ejemplo, la analítica descriptiva se puede utilizar para monitorear la participación de los estudiantes en el trabajo con el entorno digital: la frecuencia del trabajo con recursos educativos electrónicos, asistencia, datos sobre la inscripción en cursos de capacitación, etc. En presencia de dichos datos en el dominio público, los tutores y los propios estudiantes pueden monitorear la actividad de los participantes en el proceso educativo. Los modelos utilizados en el análisis predictivo, pronostican los eventos comparando las etapas de aprendizaje pasadas y actuales. Basado en tales análisis, es posible identificar a los estudiantes que son propensos a interrumpir el cronograma del proceso educativo. Además, para optimizar la trayectoria, se puede predecir cuál será el crecimiento del nivel de logros educativos bajo ciertas influencias de control. Los modelos de análisis prescriptivos se utilizan para formular recomendaciones, que puedan cambiar la trayectoria del aprendizaje. La combinación de análisis descriptivo, predictivo y prescriptivo es la base del trabajo del entorno educativo adaptativo digital.

En la Tabla 2 un ejemplo de la teoría de las zonas de desarrollo pone de relieve las características esenciales de un aprendizaje, que no pueden pasarse por alto en la transformación al entorno digital. Además, se indican las herramientas del entorno educativo necesarias para la implementación del e-learning a través de zonas de desarrollo.

Modelación matemática y algorítmica de los términos de la teoría del socioconstructivismo para un entorno educativo digital. Ivanov, I., Kosonogova, M., Cárdenas, J. 
Tabla 2. Justificación de la posibilidad de transferir la teoría del desarrollo del aprendizaje al entorno digital.

\begin{tabular}{|c|c|}
\hline Rasgo de aprendizaje del desarrollo & Realización en el entorno digital \\
\hline $\begin{array}{l}\text { La identificación de zonas de desarrollo tiene como base } \\
\text { la información sobre el volumen y la naturaleza de las } \\
\text { acciones correctivas (tipos de asistencia) requeridas para } \\
\text { resolver situaciones problemáticas. }\end{array}$ & $\begin{array}{l}\text { Un sistema de recomendación y otros tipos de } \\
\text { ayuda. Seguimiento programático de las } \\
\text { acciones de los usuarios en un entorno } \\
\text { educativo digital. }\end{array}$ \\
\hline $\begin{array}{l}\text { Evaluación oportuna y regulación de zonas de desarrollo } \\
\text { actual y próxima. }\end{array}$ & $\begin{array}{l}\text { Mecanismos de retroalimentación para } \\
\text { optimizar la ruta de aprendizaje después de } \\
\text { cada paso de aprendizaje. }\end{array}$ \\
\hline Adhesión del principio de visibilidad del entrenamiento & $\begin{array}{l}\text { Herramientas interactivas, tecnologías de } \\
\text { gamificación. }\end{array}$ \\
\hline
\end{tabular}

Nota: Elaboración propia.

\subsection{Metodología de la investigación}

La solución del problema de la construcción del modelo matemático de zonas de desarrollo se basa en la teoría de medir variables latentes y métodos de psicometría computacional. El punto de partida es la tesis de que la zona del desarrollo próxima $(Z D P)$ - la distancia entre los niveles del desarrollo actual y posible (Vygotsky 2008), es decir, podría ser descrito por el intervalo

$$
\beta_{s}<Z D P \leq \beta_{s}+\omega_{s}
$$

donde $\omega_{s}$ es un valor de umbral que caracteriza los límites de la zona (es decir anchura de zona de desarrollo próxima para $\boldsymbol{s}$-ésimo paso del entrenamiento); $\boldsymbol{\beta}_{\boldsymbol{s}}$ es nivel de capacitación en el $\boldsymbol{s}$-ésimo paso del entrenamiento.

Para comparar el ancho $\omega_{s}$ de zona del desarrollo próxima del valor numérico, se debe partir de la idea de que la zona de desarrollo es proporcional al nivel de capacitación:

$$
\frac{\omega_{s}}{\beta_{s}}=\text { coef }, \omega_{s}=\beta_{s+1}-\beta_{s} .
$$

Aquí $\boldsymbol{\beta}_{\boldsymbol{s}+\mathbf{1}}$ y $\boldsymbol{\beta}_{\boldsymbol{s}}$ son los niveles de capacitación alcanzados en los pasos de entrenamiento $(\boldsymbol{s}+\mathbf{1})$-ésimo y $\boldsymbol{s}$-ésimo; coef es un coeficiente de proporcionalidad, cuyo significado aplicado requiere aclaración más tarde.

Para evitar la relación entre el ancho de la zona de desarrollo y los pasos del entrenamiento en expresión (2) y obtener una fórmula más universal, se realizaron las siguientes acciones. Habiendo expresado el nivel de capacitación en el siguiente paso del entrenamiento a través del nivel inicial de capacitación $\boldsymbol{\beta}_{\mathbf{0}}$, obtuvimos la expresión (3).

$$
\beta_{s}=(\operatorname{coef}+1)^{s} \cdot \beta_{0}
$$

Después de la sustitución de la relación (3) a (2):

$$
\frac{\beta_{s+1}-(\operatorname{coef}+1)^{s} \cdot \beta_{0}}{(\operatorname{coef}+1)^{s} \cdot \beta_{0}}=k^{*}
$$

Expresando la variable $\boldsymbol{\beta}_{s+1}$ de (4) usando la sustitución $(\boldsymbol{c o e f}+\mathbf{1})^{s}=\boldsymbol{e}^{s \cdot \ln (\boldsymbol{c o e f}+\mathbf{1})}$ obtuvimos:

$$
\beta_{S+1}=\beta_{0} \cdot(\operatorname{coef}+1) \cdot e^{s \cdot \ln (\operatorname{coef}+1)}
$$

Modelación matemática y algorítmica de los términos de la teoría del socioconstructivismo para un entorno educativo digital. Ivanov, I., Kosonogova, M., Cárdenas, J. 
En (5) el valor de $\boldsymbol{\beta}_{\boldsymbol{s}+\mathbf{1}}$ es exponencial y se interpreta como el límite superior de la zona de desarrollo próximo en el $s$-ésimo paso de aprendizaje que no debe superarse al seleccionar los objetos del contenido del aprendizaje. En otras palabras, el nivel de complejidad de objetos de contenido presentados en el $s$-ésimo paso de aprendizaje, no debe superar el límite superior de la zona de desarrollo para esta etapa. La desventaja de la relación (5) es que el límite de la zona de desarrollo depende de la numeración de los pasos de capacitación; por lo tanto, se requiere un refinamiento del modelo. Para aclarar el modelo de la zona de desarrollo próximo, se propone utilizar las disposiciones de cálculo diferencial. Para hacer esto, introducimos las sustituciones $\boldsymbol{k}_{1}=(\boldsymbol{c o e f}+\mathbf{1}), \boldsymbol{k}_{2}=$ $\ln (\boldsymbol{c o e f}+\mathbf{1})$, con las cuales la expresión (5) toma la forma:

$$
\beta(s)=\beta_{0} \cdot k_{1} \cdot e^{k_{2} \cdot s}
$$

En las condiciones de la numeración aceptada de los pasos de aprendizaje, la anchura de la zona del desarrollo próximo en $s$-ésimo paso puede ser descrita en la forma de la relación

$$
\omega_{s}=\frac{\beta_{s+1}-\beta_{s}}{(s+1)-s} .
$$

Haciendo la transición en límite

$$
\omega=\lim _{\Delta s \rightarrow 0} \frac{\Delta \beta}{\Delta s}
$$

e interpretando $\omega$ como la derivada de $\beta(s)$, obtenemos:

$$
\omega=\frac{d \beta(s)}{d s}=\beta_{0} \cdot k_{1} \cdot k_{2} \cdot e^{k_{2} \cdot s}
$$

En la diferenciación repetida de (6) tenemos:

$$
\frac{d \omega}{d s}=\beta_{0} \cdot k_{1} \cdot k_{2}^{2} \cdot e^{k_{2} \cdot s}=k_{2} \cdot \omega
$$

Con base a la posición sobre la proporcionalidad del diferencial de la función al incremento del argumento de esta función, escribimos:

$$
\frac{d \omega}{d s} \cdot \frac{d s}{d \beta}=\omega \cdot k_{3} \cdot e^{-k_{2} \cdot s},
$$

donde $\boldsymbol{k}_{3}=\boldsymbol{\beta}_{0} \cdot \boldsymbol{k}_{1}$

Suponiendo que el parámetro $s$ en (7) es fijo, introducimos de una sustitución de componentes $\boldsymbol{k}_{\mathbf{3}}=\boldsymbol{\beta}_{\mathbf{0}} \boldsymbol{k}_{\boldsymbol{1}}$ por un factor de proporcionalidad $\boldsymbol{C}$. Entonces, tenemos una ecuación diferencial:

$$
\frac{d \omega}{d \beta}=C \cdot \omega
$$

La solución general de la ecuación diferencial (8) será la siguiente:

$$
\omega=\omega_{0} \cdot e^{C \cdot \beta},
$$

Modelación matemática y algorítmica de los términos de la teoría del socioconstructivismo para un entorno educativo digital. Ivanov, I., Kosonogova, M., Cárdenas, J. 
donde $\omega_{0}$ es anchura de la zona de desarrollo próximo con nivel medio de la capacitación, por lo que en la teoría de los rasgos latentes se supone $\boldsymbol{\beta}=\mathbf{0}$ (logit, una medida del nivel de capacitación); $\boldsymbol{C}$ es la constante que determina la tasa de crecimiento de la zona de desarrollo próximo.

En la teoría de medición de los rasgos latentes (Maslak 2016, Rasch 1980), se utilizan dos medidas, presentadas utilizando una escala logarítmica: el logit del nivel de capacitación y el logit del nivel de complejidad de la tarea (material, objeto de contenido). Aumentar el nivel de capacitación por un logit significa un aumento en la unidad del logaritmo de la razón de la probabilidad de la respuesta correcta de prueba a la probabilidad de la incorrecta.

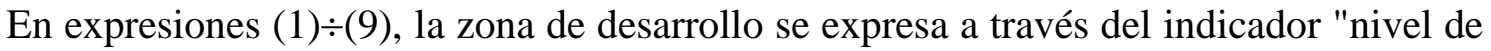
capacitación", cuyo valor numérico es necesario para calcular los límites de la zona de desarrollo próximo. Se conocen enfoques generales para la determinación cuantitativa del nivel de capacitación (Maslak 2016). La idea de que la capacitación en forma explícita no puede evaluarse ni medirse, da razones para considerarla como una variable latente. Para desarrollar el aprendizaje en el entorno digital, es importante que el modelo de competencias tenga en cuenta el patrón de interacción del usuario con el sistema de aprendizaje y el uso de los mensajes en el entorno digital. Desde el punto de vista de la psicometría computacional (Abbakumov, Desmet, \& Van den Noortgate 2018, Wilson \& Nichols 2015), el patrón de interacción se describe en forma de una matriz de datos: una "huella digital", que incluye para cada usuario: estadísticas sobre el paso de las tareas de prueba; la dinámica de los cambios en los indicadores de actividades educativas; estadísticas de interacción con contenido y una plataforma de aprendizaje; niveles de desarrollo de funciones cognitivas; indicadores de estado funcional en el proceso de aprendizaje etc. Por lo tanto, los datos de la "huella digital" son indicadores del nivel de capacitación. Entonces, para una descripción formal del nivel de capacitación, se propone una lista de indicadores medibles de la tabla 3.

Tabla 3. Métricas de interacción del usuario con el entorno educativo digital.

\begin{tabular}{|c|c|}
\hline Símbolo & \multicolumn{1}{|c|}{ Descifrado del indicador } \\
\hline$y_{2,1}$ & número de omisiones de prueba \\
\hline$y_{2,2}$ & número de opciones de cambio de respuesta dentro de la prueba \\
\hline$y_{2,3}$ & tiempo para el uso al contenido educativo durante el control de la prueba \\
\hline$y_{2,4}$ & profundidad de visualización de la estructura del contenido educativo al pasar el examen \\
\hline$y_{2,5}$ & visualización los fragmentos multimedia que se ofrecen para aprender contenido \\
\hline$y_{2,6}$ & inacción del sistema \\
\hline$y_{2,7}$ & tasa de fallos de la prueba \\
\hline$y_{2,8}$ & número de pasos para los cuales se completó la etapa de aprendizaje \\
\hline $\mathrm{y}_{2,9}$ & número de los usos el software externo al pasar el control de prueba \\
\hdashline $\mathrm{y}_{2,10}$ & tiempo necesario para pasar la prueba \\
\hline $\mathrm{y}_{2,11}$ & tiempo dedicado a aprender los bloques de contenido educativo \\
\hline $\mathrm{y}_{2,12}$ & número de los usos del glosario mientras se aprendía contenido \\
\hdashline $\mathrm{y}_{2,13}$ & número de los usos del sistema de pistas durante de estudio el contenido educativo. \\
\hdashline $\mathrm{y}_{2,14}$ & número de los usos otros recursos durante el estudio del contenido educativo. \\
\hline
\end{tabular}

Nota: Elaboración propia.

Modelación matemática y algorítmica de los términos de la teoría del socioconstructivismo para un entorno educativo digital. Ivanov, I., Kosonogova, M., Cárdenas, J. 
Las métricas de interacción del usuario con la plataforma se fijan para cada etapa $s$-ésimo junto con los resultados de las pruebas. Generalmente, el cortejo de indicadores para el modelo de capacitación es $\left\langle\boldsymbol{y}_{1,1} \ldots \boldsymbol{y}_{1, k}, \boldsymbol{y}_{2,1} \ldots \boldsymbol{y}_{2, l}\right\rangle$, donde $\boldsymbol{y}_{1,1} \ldots \boldsymbol{y}_{1, k}$ es un conjunto de indicadores con los resultados de $\boldsymbol{k}$ tareas de control, $\boldsymbol{y}_{2, \boldsymbol{l}} \ldots \boldsymbol{y}_{2, l}$ es un conjunto de $\boldsymbol{l}$ factores de comportamiento según la Tabla 3)

Los datos del siguiente paso de aprendizaje se muestrean y procesan utilizando un modelo IRT (Item Response Theory (inglés) o Teoría de Respuesta al Ítem, Teoría del Rasgo Latente) adecuado (dicotómico o politómico), que se incluye en el aparato de medición de la teoría de rasgos latentes. Los modelos de IRT difieren en el tipo de la función $\boldsymbol{P}(\boldsymbol{x})$, que se introduce para estimar la probabilidad de éxito del estudiante $\boldsymbol{i}$-ésimo en la realización de tareas. Dado que los factores de comportamiento considerados (de la Tabla 3) pueden tomar más de dos valores, el modelo del nivel de capacitación puede ser descrito mediante la siguiente dependencia politómica (Maslak 2012):

$$
P\left\{x_{i j}=x\right\}=\frac{e^{-\tau_{1 j^{-}} \tau_{2 j^{-\cdots-\tau}} \tau_{x j}+x \cdot\left(\beta_{i}-\delta_{j}\right)}}{\sum_{x^{\prime}=0}^{m_{j}} e^{-\tau_{1 j^{-}}-\tau_{2 j^{-\cdots}-\tau_{x j}+x^{\prime} \cdot\left(\beta_{i}-\delta_{j}\right)}}}
$$

Aquí $\boldsymbol{x}$ es la graduación de la variable indicadora obtenida en base a la discretización ; $\boldsymbol{x}_{i j}$ es respuesta del estudiante $\boldsymbol{i}$-ésimo al indicador $\boldsymbol{j}$-ésimo; $\boldsymbol{P}\left\{\boldsymbol{x}_{i j}=\boldsymbol{x}\right\}$ es probabilidad de que el estudiante $\boldsymbol{i}$-ésimo seleccione la opción $\boldsymbol{x}$ del indicador $\boldsymbol{j}$-ésimo; $\boldsymbol{\delta}_{\boldsymbol{j}}$ es el nivel de complejidad de la variable indicadora; $\tau_{x j}$ es nivel relativo de complejidad de graduación $\boldsymbol{x}$-ésimo del indicador $\boldsymbol{j}$-ésimo entendido como un incremento del nivel capacitación necesario para la transición entre las graduaciones; $\boldsymbol{m}_{\boldsymbol{j}}$ es una variable de índice que acepta todas las opciones del indicador $\boldsymbol{j}$-ésimo.

Aceptamos que en todas las regularidades que describen los parámetros de la zona de desarrollo próximo, el nivel de capacitación se mide sobre la base de (10) teniendo en cuenta el nivel de dificultad de las tareas a resolver, el factor de utilizar diferentes tipos de ayuda y otras interacciones del usuario con el entorno de aprendizaje.

Para la selección de los coeficientes $\omega_{0}$ y $\boldsymbol{C}$ de expresión (9) se ofrece el algoritmo de ocho pasos.

Algoritmo 1. Selección de los parámetros de la zona de desarrollo próximo:

1. Preparar herramientas de evaluación con un nivel conocido de complejidad $\boldsymbol{\delta}$ para medir el nivel de capacitación de un grupo de sujetos. La validez y la fiabilidad de las herramientas de evaluación deben verificarse para excluir las tareas de prueba extremadamente difíciles o fáciles, así como para equilibrar el conjunto de tareas con la porción de pruebas de diferentes niveles de dificultad.

2. Medir el nivel de capacitación $\beta$ y posponer los resultados en una escala única en los logits, que se adopta en el marco de la teoría de medición de los rasgos latentes. Para medir el nivel de capacitación se desarrolla el sistema de ecuaciones (10) para el conjunto de estudiantes, que se resuelve con respecto a $\beta_{i}$ de tal manera que describa la matriz con los resultados de la prueba y los valores de los factores de comportamiento con la mayor precisión posible. $\boldsymbol{P}\left\{\boldsymbol{x}_{\boldsymbol{i} \boldsymbol{j}}=\boldsymbol{x}\right\}$

3. Seleccionar dos puntos en la vecindad de cero en la escala del nivel de capacitación, que forman el rango $\left[-\beta^{*} ;+\beta^{*}\right]$ con la mayor frecuencia de los resultados de medición $\beta$.

Modelación matemática y algorítmica de los términos de la teoría del socioconstructivismo para un entorno educativo digital. Ivanov, I., Kosonogova, M., Cárdenas, J. 
4. Seleccionar un punto de referencia adicional con un nivel de capacitación aleatorio $\boldsymbol{\beta}_{\text {add }}$.

5. Seleccionar $n$ estudiantes con nivel de capacitación $\boldsymbol{\beta}_{i} \approx \boldsymbol{\beta}_{\text {add }}(\boldsymbol{i}=\mathbf{1} \ldots \boldsymbol{n})$.

6. Presentar consistentemente a un grupo de $\boldsymbol{n}$ sujetos tareas de nivel de dificultad creciente (el nivel inicial de dificultad $\boldsymbol{\delta}$ debe ser igual a $\boldsymbol{\beta}_{\text {add }}$ ).

7. Registrar el valor del nivel de complejidad $\boldsymbol{\delta}_{\text {lim }}$, en el que $93-95 \%$ del grupo de sujetos no puede completar con éxito las tareas. Para cumplir con el concepto de desarrollo del aprendizaje, las pruebas deben llevarse a cabo en condiciones en las que esté disponible un sistema de ayuda y pistas.

8. Encontrar los coeficientes $\omega_{0}, \boldsymbol{C}$ solucionando el sistema de ecuaciones

$$
\left\{\begin{array}{c}
\omega_{0} \cdot e^{-\beta^{*} \cdot C} \cdot 1.2=\omega_{0} \cdot e^{+\beta^{*} \cdot C}, \\
\omega_{0} \cdot e^{\delta_{0} \cdot C}=\delta_{\text {lim }}-\delta_{0},
\end{array}\right.
$$

donde 1.2 es un coeficiente tomado de consideraciones didácticas, que, con el nivel de capacitación alcanzado, el sujeto tiene potencialmente en promedio un $20 \%$ más de dificultad para dominar el material.

La segunda ecuación en el sistema (11) se hace a partir de consideraciones de que las tareas con nivel de complejidad $\boldsymbol{\delta}_{\boldsymbol{0}}<\boldsymbol{\delta} \leq \boldsymbol{\delta}_{\text {lim }}$ (logits) están en la zona de desarrollo próximo de cada estudiante $i$-ésima del grupo con $\boldsymbol{\beta}_{\boldsymbol{i}} \approx \boldsymbol{\beta}_{\text {add }}(i=1 . . n)$. La aplicación de la teoría de rasgos latentes permite considerar los parámetros $\boldsymbol{\beta}^{*}, \boldsymbol{\omega}_{\mathbf{0}}, \boldsymbol{\delta}_{\mathbf{0}}$ y $\boldsymbol{\delta}_{\text {lim }}$ de este sistema de ecuaciones en la escala única en logits.

\subsection{Resultados prácticos}

La implementación del modelo de la zona de desarrollo próximo nos permite ampliar el modelo digital del alumnado en términos de tener en cuenta las características individuales que afectan el éxito de la formación. Por lo tanto, es más apropiado considerar la aplicación del modelo pedagógico construido para la gestión adaptativo del e-learning (Figura 2). El algoritmo presentado es una generalización del algoritmo de control de trayectoria educativa (Kosonogova 2016) ampliando los métodos de búsqueda para optimizar la trayectoria.

Los bloques desde 1 hasta 8 en la Figura 2 están destinados a introducir en el circuito de control del desarrollo del aprendizaje los parámetros individuales del estudiante: el nivel de capacitación y las características de la zona de desarrollo próximo. En la etapa preparatoria, se determina el nivel actual de logros educativos para inicializar los parámetros del modelo digital del alumnado (Figura 2, bloque 1). Con base a esto, se toma una decisión sobre el punto de partida de la trayectoria de aprendizaje (Figura 2, bloque 2 ). Además, en cada paso $s$, se realizan los siguientes procedimientos hasta que se alcanza el objetivo de aprendizaje. Se monitorean las salidas medidas del objeto de control. Los indicadores de actividad educativa y los indicadores de comportamiento de los usuarios del entorno educativo digital son objetos de monitoreo (Figura 2, bloque 4). Como indicadores de comportamiento se pueden tomar factores de la tabla. 3. Sobre la base de los valores recopilados de los indicadores, se mide el nivel actual de capacitación $\boldsymbol{\beta}_{\boldsymbol{s}}$ (Figura 2, bloque 5). Los cálculos se hacen a partir de aquellas consideraciones de que la probabilidad de éxito del entrenamiento para cada indicador se describe por la relación (10). En el bloque 6 (Figura 2), se implementa una verificación de la lógica del desarrollo de la educación. A saber, en las relaciones (1) y (9), se revela si se domina la zona de

Modelación matemática y algorítmica de los términos de la teoría del socioconstructivismo para un entorno educativo digital. Ivanov, I., Kosonogova, M., Cárdenas, J. 
desarrollo próximo. Si se registra que el material pasó a la zona de desarrollo actual, entonces tiene sentido volver a calcular los parámetros de la zona de desarrollo proximal (Figura 2, bloque 7). El recálculo de los parámetros de la zona de desarrollo próximo significa que la etapa de entrenamiento anterior se completó con éxito y se logró un incremento en el nivel de capacitación. De lo contrario, se aceptan los parámetros de la zona de desarrollo calculados en el paso anterior de entrenamiento (Figura 2, bloque 8). La lógica básica para tomar de decisiones sobre la formación de paso $s$-ésimo fragmento $\boldsymbol{u}_{s}$ de trayectoria se establece en los bloques 10-13. El bloque 12 contiene una implementación del método de optimización de un solo paso o de varios pasos. Por ejemplo, con diferentes enfoques, los siguientes valores se pueden tomar como el criterio para la optimización del proceso educativo:

- tiempo para estudiar material didáctico;

- nivel de conocimiento, destrezas y/o habilidades;

- tamaño del bloque de información de entrenamiento;

- nivel de carga cognitiva de acuerdo con el estado funcional actual del estudiante;

- la cantidad de información recibida y adquirida por el estudiante como la diferencia de entropías. 
RED. Revista de Educación a Distancia. Núm. 64, Vol. 20. Artíc. 7, 30-09-2020

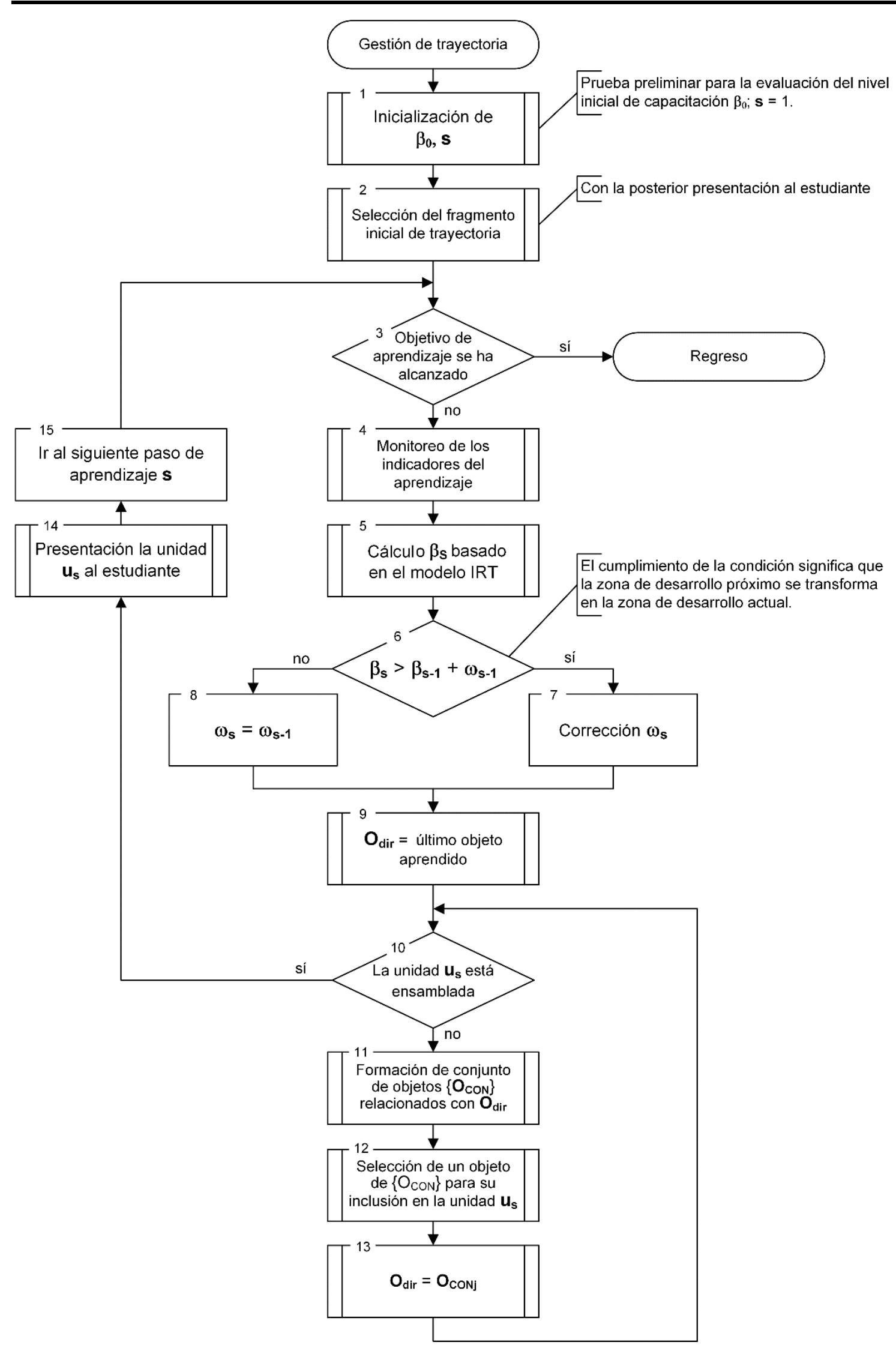

Figura 2. Algoritmo de la gestión adaptativa del proceso de desarrollo del aprendizaje.

En el marco de la teoría del desarrollo del aprendizaje, esta lista de criterios de optimalidad puede ampliarse con los parámetros de las zonas de desarrollo. Luego, para aumentar la adaptabilidad y la personificación del proceso de gestión, se puede centrar la Modelación matemática y algorítmica de los términos de la teoría del socioconstructivismo para un entorno educativo digital. Ivanov, I., Kosonogova, M., Cárdenas, J. 
atención en la probabilidad de iteración errónea del proceso del aprendizaje. Esto se refiere a la probabilidad de una transición errónea a la zona de desarrollo posterior o viceversa, a la zona de desarrollo actual, debido a la inclusión de un bloque de información de la trayectoria individual. Teniendo en cuenta la teoría aceptada del desarrollo de aprendizaje, para desarrollar la acción de control $\boldsymbol{u}_{\boldsymbol{s}}$ se lleva a cabo tomando en consideración la zona formal de desarrollo próximo. En este caso, el desarrollo de la influencia de gestión se entiende como la formación adaptativa de un bloque de objetos de contenido, que asegura la adaptación del material presentado de acuerdo con el nivel de complejidad y el nivel de cumplimiento de los parámetros individuales de la zona de desarrollo del estudiante. Generalmente, los métodos de gestión de la trayectoria de aprendizaje adaptativo le permiten organizar canales de retroalimentación en un entorno educativo digital que garantizan la corrección del proceso de aprendizaje teniendo en cuenta muchos aspectos: el potencial de los estudiantes asociados con sus características personales y psicológicas; logros de aprendizaje y factores de comportamiento; condición funcional.

Se propone la siguiente metodología para evaluar la viabilidad de aplicar el modelo de la zona de desarrollo próximo. Como material experimental, se utilizan datos sobre los resultados de la actividad educativa de $N$ sujetos, organizados en forma de una matriz bidimensional $\boldsymbol{Y}$ de dimensión $N \times(\boldsymbol{k}+\boldsymbol{l})$. Las mediciones matriciales son los indicadores probados y registrados de la actividad de aprendizaje del corteje $\left\langle\boldsymbol{y}_{1,1} \ldots \boldsymbol{y}_{1, k}, \boldsymbol{y}_{2,1} \ldots \boldsymbol{y}_{2, l}\right\rangle$, donde $\boldsymbol{y}_{1,1} \ldots \boldsymbol{y}_{1, \boldsymbol{k}}$ es un bloque de indicadores con los resultados de $\boldsymbol{k}$ tareas de prueba, $\boldsymbol{y}_{2,1} \ldots \boldsymbol{y}_{2, l}$ es un bloque de $\boldsymbol{l}$ factores de comportamiento de acuerdo con la Tabla 3. Si es imposible registrar cualquier factor de comportamiento en el proceso de e-learning, se excluyen de la consideración. Los resultados de las actividades educativas deben ordenarse por temas o secciones de la disciplina estudiada. La siguiente hipótesis está sujeta a verificación: el nivel de capacitación de la mayoría de los estudiantes alcanzado durante la capacitación para el desarrollo es un cierto valor más alto que los resultados del e-learning sin tener en cuenta la zona de desarrollo próximo.

Algoritmo 2. Estimación de la conveniencia de aplicar el modelo de la zona de desarrollo proximal.

1. Cálculo basado en un sistema de ecuaciones (10) de niveles de capacitación $\boldsymbol{\beta}_{\boldsymbol{i}}$ todos los estudiantes $(i=1 \ldots N)$ y niveles de complejidad $\delta_{j}$ todos los indicadores $(j=1 \ldots k+l)$ basados en los resultados de un estudio de todos los temas de la materia.

2. Preparación para la simulación del proceso de aprendizaje del desarrollo. El nivel inicial de capacitación de cada sujeto $\boldsymbol{\beta}_{z}{ }^{(0)}(z=1 \ldots N)$ se calcula con la base de los resultados de estudiar el primer tema de la materia.

3. Realización los pasos del algoritmo que se muestra en la Figura 2 para cada sujeto. En el bloque 4 se genera los indicadores de actividad educativa en lugar de monitorearlos. Para esto, se selecciona una fila de la matriz original $\boldsymbol{Y}$ por la cual $\boldsymbol{\beta}_{\boldsymbol{i}} \approx \boldsymbol{\beta}_{z^{(\boldsymbol{)})}}(\boldsymbol{i} \neq \boldsymbol{z})$. El objetivo de esta acción es determinar el modelo de comportamiento del usuario, lo que conduce al logro de un nivel fijo de capacitación. Este punto se repite hasta que se agoten todos los bloques de materia. Como resultado, el nivel de capacitación del sujeto después de los pasos de entrenamiento alcanza $\boldsymbol{\beta}_{z}{ }^{(s)}$.

4. Comparación de los niveles de capacitación para cada sujeto de simulación. Confirmación o refutación de la hipótesis.

Para justificar las conclusiones teóricas del uso del modelo de zonas de desarrollo (9) a lo de aprendizaje adaptativo (según el algoritmo de la figura 2), se utilizó los siguientes

Modelación matemática y algorítmica de los términos de la teoría del socioconstructivismo para un entorno educativo digital. Ivanov, I., Kosonogova, M., Cárdenas, J. 
datos experimentales: 1) Resultados del aprendizaje física de 310 estudiantes de la Universidad Estatal de Milagro (Ecuador); 2) los resultados de la capacitación en informática de 1159 estudiantes de la Universidad Estatal Tecnológica de Belgorod (Rusia). En el primer caso, el control de estudio se llevó a cabo sobre la base de 4 bloques de prueba en línea para 10 problemas y un indicador de comportamiento a saber el tiempo de prueba. En el segundo caso, basado en 4 bloques de materia, incluyendo 60 ítems de prueba y contenido teórico, y además los indicadores de comportamiento de la tabla 3 . En cada caso los valores de los indicadores registrados durante el monitoreo del software, se procesaron utilizando el modelo IRT (10) mediante el software RUMM 2020. Los rangos obtenidos de niveles de dificultad $\boldsymbol{\delta}_{j}$ de tareas y niveles de capacitación $\boldsymbol{\beta}_{\boldsymbol{i}}$ de estudiantes necesitaba para la selección experimental de parámetros del modelo de la zona de desarrollo próximo. Por sede de la Universidad Estatal de Milagro están fijado los rangos de $\delta \in[-3,1 ; 2,5]$ y $\beta \in[-2,7 ; 4,0]$ logits, y en el sitio de la Universidad Estatal Tecnológica de Belgorod se obtuvieron los valores de $\delta \in[-4,8 ; 6,5]$ y $\boldsymbol{\beta} \in[-5,0 ; 6,0]$ logits. Sobre la base del Algoritmo 1 y los datos presentados, se obtuvieron los siguientes parámetros del modelo (9): Milagro: $\boldsymbol{\omega}_{\mathbf{0}}=\mathbf{0 . 3 0}, \mathbf{C}=\mathbf{0 . 2 4}$; Belgorod: $\boldsymbol{\omega}_{\mathbf{0}}=\mathbf{0 . 2 7}, \mathbf{C}=\mathbf{0 . 2 6}$. La proximidad de los conjuntos de parámetros puede explicarse por el hecho de que en ambos sitios se utilizaron principalmente los resultados de las actividades de capacitación de estudiantes medianamente entrenados. Aceptamos las diferencias entre los conjuntos de parámetros como insignificantes, ya que la secuencia de valores del ancho de la zona de desarrollo proximal se obtuvo para el rango $\beta \in[-\mathbf{6 , 0} \mathbf{0} \mathbf{6 , 0}]$ logits, tienen estimaciones cercanas de las esperanzas matemáticas $(\mathbf{m})$ y variaciones $(\mathbf{D})$, a saber: Milagro: $\boldsymbol{m}=$ 0.4052, $D=0.0047$; Belgorod: $\boldsymbol{m}=\mathbf{0 . 4 2 5 6}, D=0.0050$.

La consistencia del modelo de la zona de desarrollo próximo se verificó sobre la base de los datos del sitio de la Universidad Estatal de Milagro. Basado en el modelo de simulación del proceso de aprendizaje del desarrollo (de acuerdo con la Figura 2, con los parámetros de la zona de desarrollo $\omega_{\mathbf{0}}=\mathbf{0 . 3 0}, \mathbf{C}=\mathbf{0 . 2 4}$ ), se encontró que para el $\mathbf{5 9 \%}$ de los estudiantes de la muestra inicial ( $\mathrm{N}=92$ personas), es posible un incremento del nivel final preparación del 4.3-6.7\%. El efecto se logra presentando en el próximo paso de entrenamiento tal tareas de prueba, cuyo nivel de complejidad es comparable al nivel de capacitación alcanzado. La calificación obtenida puede mejorarse, en primer lugar, mediante el uso de un modelo ampliado para monitorear los resultados académicos. Esto se refiere al cálculo del nivel de preparación según el modelo (Ecuación 10) teniendo en cuenta el conjunto de indicadores de comportamiento. En segundo lugar, para el aprendizaje del desarrollo, se deberían utilizar herramientas de evaluación cubriendo una amplia gama de cambios en el nivel de capacitación. En otras palabras, debe excluirse la situación (ver Figura 3) en la que el nivel de dificultad de las tareas de evaluación se concentra en un rango estrecho de la escala de medición.

Modelación matemática y algorítmica de los términos de la teoría del socioconstructivismo para un entorno educativo digital. Ivanov, I., Kosonogova, M., Cárdenas, J. 


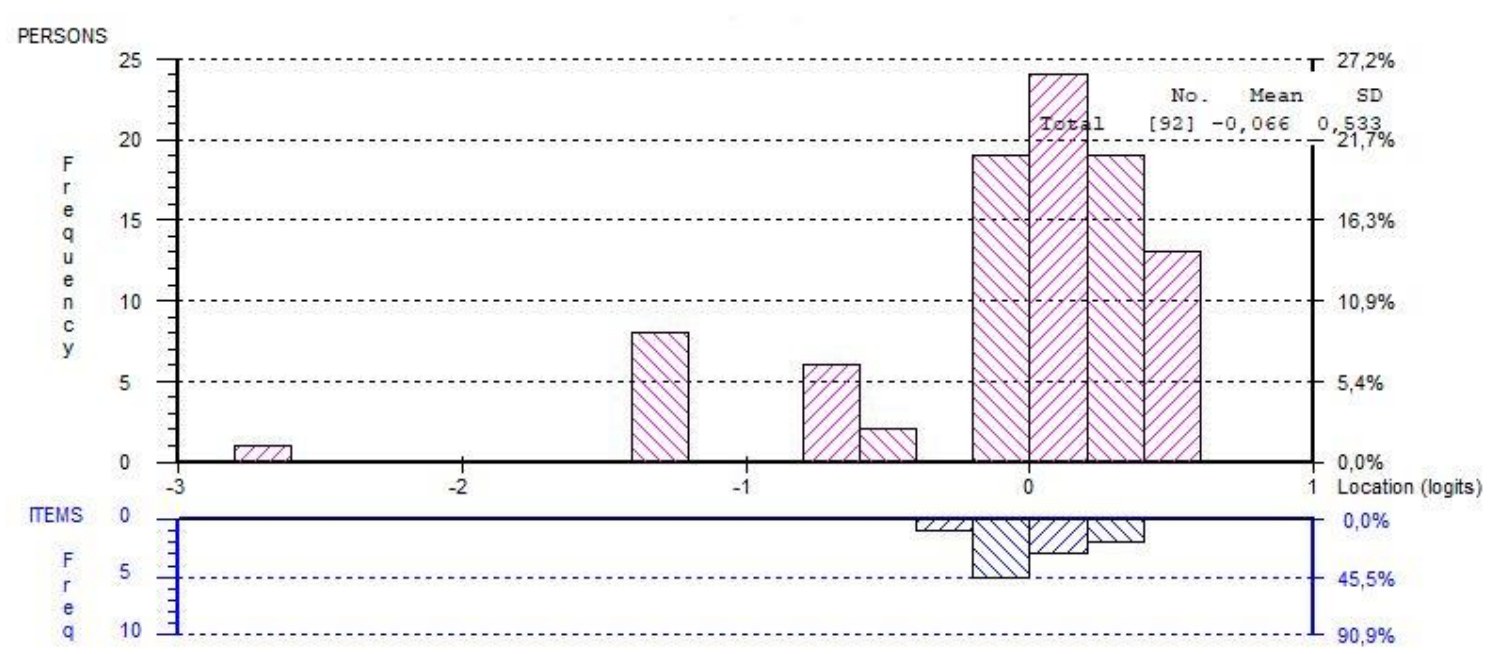

Figura 3. Distribución de los niveles de capacitación (arriba) y la complejidad de la tarea (abajo) en un experimento computacional en la Universidad Estatal Milagro.

\subsection{Enfoques a la arquitectura del entorno educativo moderno digital.}

Las tendencias y herramientas tecnológicas facilitan la implementación de modelos y métodos de enseñanza al entorno digital de una arquitectura abierta. Por lo tanto, desde un punto de vista técnico, las siguientes tendencias deben tenerse en cuenta al construir entornos educativos digitales modernos:

- desarrollo de proyectos educativos basados en la infraestructura de la nube;

- estandarización de las funciones API para la interacción de diferentes servicios educativos entre sí y la implementación de la idea de ecosistemas de aprendizaje abierto;

- ampliación de las oportunidades del sistema de entrenamiento a través de nuevos componentes (ver Tabla 1), así como conectar asistentes de voz (Siri), bots de chat, simuladores cognitivos (BrainHQ, NeuroNation, Wikium) y servicios de seguimiento de emociones (Emotuit);

- la acumulación de datos, que es una "huella digital" de estudiantes, en el paradigma de Big Data y su análisis basado en el aprendizaje automático, los sistemas de recomendación y otras tecnologías de inteligencia artificial;

- desarrollo de estándares y herramientas técnicas para el registro abierto de los logros en línea (es decir, creación, emisión, gestión y validación los certificados de aprendizaje en línea), recogidas de diferentes recursos y plataformas educativos.

En los párrafos 2.1 a 2.3 describen y justifican número de soluciones conceptuales para el modelado y automatización de procesos psicológicos y pedagógicos en el entorno digital, a saber:

- se fundamentó la formación en línea en forma de una teoría del desarrollo del aprendizaje;

- adoptó un esquema general de interacción de estudiantes con el sistema basado en el enfoque cibernético;

- se propuso un modelo de apoyo para el sistema de formación como parte del modelo matemático de la zona de desarrollo (Ecuación 9);

- se ha desarrollado un método para gestionar el proceso de aprendizaje (Figura 2) con criterios formalizados para optimizar y adaptar las trayectorias educativas.

Para implementarlas, es necesario elegir la arquitectura típica del sistema de información y adaptarla a las tareas del aprendizaje electrónico individualizado. La arquitectura

Modelación matemática y algorítmica de los términos de la teoría del socioconstructivismo para un entorno educativo digital. Ivanov, I., Kosonogova, M., Cárdenas, J. 
monolítica, que combina el nivel de representación de datos, el nivel de lógica de negocio y el nivel de almacenamiento de datos en un solo entorno físico, y la arquitectura de "servidor de archivos", que implica la introducción de un servidor de base de datos dedicado, están fuera del alcance debido a la dificultad de escalado. Para el despliegue de un entorno educativo moderno digital, son más apropiados:

- una arquitectura "cliente-servidor" de tres niveles: cliente de la aplicación, el servidor de aplicaciones y el servidor de bases de datos, pueden ubicarse en diferentes computadoras;

- una arquitectura de warehouse (almacenes de datos), que incluye una base de datos analítica, que recopile datos de bases de datos operativas y fuentes de datos externas, bajo el control de un sistema de procesamiento de información analítica;

- una arquitectura de aplicación distribuida integrada que describe los servicios de acceso a una base de datos distribuida que combina las bases de datos de tipos diferentes.

Las arquitecturas se enumeraron en orden de oportunidades crecientes para el escalado horizontal y vertical del sistema, así como el escalado a nivel de las funciones comerciales.

La Figura 4, muestra la arquitectura del entorno educativo digital obtenido al proyectar funciones de gestión del aprendizaje en una arquitectura típica de cliente-servidor de tres niveles. El lado del servidor de la plataforma educativa se puede implementar como servicios Service-Oriented Architecture (Arquitectura Orientada a Servicios, SOA), es decir en forma de componentes distribuidos con interfaces estandarizadas para la interacción con el ecosistema educativo.

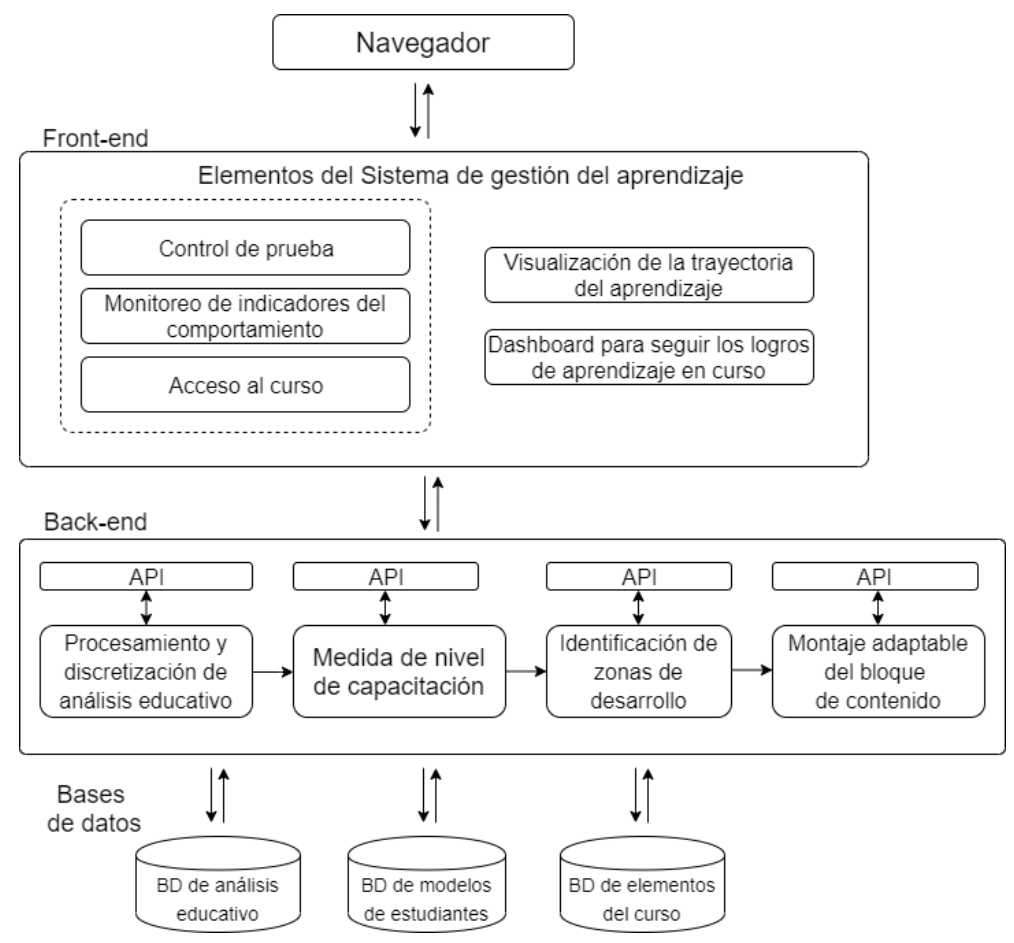

Figura 4. Arquitectura "cliente-servidor" del entorno educativo digital.

Para una descomposición detallada de cada uno de los niveles de la arquitectura presentada es necesario elaborar separadamente:

- mecanismos para monitorear indicadores de comportamiento y recopilar datos de estudiantes;

Modelación matemática y algorítmica de los términos de la teoría del socioconstructivismo para un entorno educativo digital. Ivanov, I., Kosonogova, M., Cárdenas, J. 
- arquitectura física del entorno educativo digital con la configuración de componentes de software;

- pila de tecnología para la implementación de cada nivel;

- una API para la interactuación con servicios que implementan modelos y métodos de aprendizaje electrónico adaptativos.

\section{Conclusión}

El artículo considera el proceso de transferencia de la teoría científica de socioconstructivismo al entorno educativo digital. Como resultado, obtuvimos la dependencia matemática (Ecuación 9), que nos permite expresar numéricamente el ancho de la zona de desarrollo próximo. Se propone un algoritmo para la selección experimental de parámetros del modelo de zona de desarrollo próximo, conociendo el nivel de capacitación de los alumnados y también el nivel de complejidad de las herramientas de evaluación. Al mismo tiempo, el uso del modelo IRT aumenta la flexibilidad y la variabilidad del proceso de medición del nivel de capacitación ya que entornos educativos digitales pueden utilizar un conjunto de indicadores diferentes de la actividad educativa, los que se procesan fácilmente utilizando las herramientas de la teoría de rasgos latentes. El modelo de la zona de desarrollo (Ecuación 9) se incorporó al algoritmo de gestión adaptativa del proceso de desarrollo del aprendizaje (Figura 2). Nuestras futuras investigaciones posibles, estarán relacionadas con la construcción de una familia completa de modelos de la zona de desarrollo próximo. La relevancia de esta tarea se justifica por el hecho de que los parámetros y la estructura del modelo de enseñanza en desarrollo pueden variar según el grupo de edad, el tipo de temperamento, el nivel de desarrollo de las funciones cognitivas y otras características.

Además, se considera las arquitecturas típicas de los sistemas de información de la esfera de educación. Se destacan las arquitecturas que cumplen con los requisitos técnicos y conceptuales para el digital entorno educativo moderno. Se describe la inmersión de la teoría del aprendizaje del desarrollo en un entorno "cliente-servidor".

Dado que las zonas de desarrollo se relacionan con la personalidad y las características psicológicas del individuo, el uso del modelo ZDP en la organización del aprendizaje electrónico puede mejorar la adecuación de la modelación de procesos psicológicos y pedagógicos en un entorno digital. Esta hipótesis se confirmó en el marco de la simulación del proceso de aprendizaje del desarrollo (Algoritmo 2). En otras palabras, el modelo de la zona de desarrollo puede tomarse como base para el método de gestión del proceso de aprendizaje, el método de formación de influencias educativas y de control, así como el modelo de adaptación.

Presentación del manuscrito: 1 de abril de 2019

Fecha de aprobación: 23 de junio de 2020

Fecha de publicación: 30 de septiembre de 2020

Ivanov, I., Kosonogova, M., y Cárdenas, J. (2020). Modelación matemática y algorítmica
de los términos de la teoría del socioconstructivismo para un entorno educativo
digital. RED. Revista Educación a Distancia, 20(64).
http://dx.doi.org/10.6018/red.409761

\section{Financiación}

Esta investigación no ha recibido ninguna subvención específica de los organismos de financiación en los sectores públicos, comerciales o sin fines de lucro.

Modelación matemática y algorítmica de los términos de la teoría del socioconstructivismo para un entorno educativo digital. Ivanov, I., Kosonogova, M., Cárdenas, J. 


\section{Referencias bibliográficas}

Abbakumov, D., Desmet, P. \& Van den Noortgate, W. (2018). Measuring student's proficiency in MOOCs: multiple attempts extensions for the Rasch model. Heliyon, 4(12), 1-15. DOI: 10.1016/j.heliyon.2018.e01003

Birjalia, M., Beni-Hssanea, A. \& Erritalib, M. (2018). A novel adaptive e-learning model based on Big Data by using competence-based knowledge and social learner activities. Applied Soft Computing, 69, 14-32. DOI: 10.1016/j.asoc.2018.04.030

Bodrova, E., \& Leong, D.J. (2007). Tools of the mind. The Vygotskian approach to early childhood education. Englewood Cliffs (NJ): Merrill/Prentice Hall.

Bosco, E.B. (2013). Los límites de la escuela. Educación, desigualdad y aprendizajes. México: El Colegio de Mexico AC.

Ferreyra, H.A. \& Pedrazzi, G. (2007). Teorías y enfoques psicoeducativos del aprendizaje. Buenos Aires: Noveduc Libros.

Hakkarainen, P. \& Korepanova, I. (2009). The status of the Vygotsky's «Zone of proximal development» in Russian psychology today. Journal of Russian \& east European psychology, 47 (6), 3-8.

Kosonogova, M. (2016). Mетодbl и средства управления образовательной траекторией в системах электронного обучения (Métodos y herramientas de gestión de la trayectoria educativa en los sistemas de e-learning) - Tesis doctoral. Kursk, Rusia: La Suroeste Universidad Estatal (en ruso).

Lazo, C., Osuna-Acedo, S. \& Quintana, J. (2019). sMOOC: A pedagogical model for social inclusión. Heliyon, 5(3), e01326. DOI: 10.1016/j.heliyon.2019.e01326

Lin, H.-Y., Tseng, S.-S., Weng, J.-F. \& Su, J.-M. (2009). Design and Implementation of an Object Oriented Learning Activity System. Educational Technology \& Society, $12(3), 248-265$.

Maslak, A. (2016). Теория и практика измерения латентных переменных в образовании (Teoría y práctica de medición de rasgos latentes en educación). Moscú: Urait (en ruso).

Maslak, A. (2012). Измерение латентных переменных в социальных системах (Medición de rasgos latentes en sistemas sociales). Slavyansk-on-Kuban: Centro editorial de KubSU (en ruso).

Pozo, I.J., Scheuer, N., Pérez, M., Mateus, M. \& Martín, E. (2006). Nuevas formas de pensar la enseñanza y el aprendizaje: Las concepciones de profesores y alumnos. Barcelona: Grao.

Rasch, G. (1980). Probabilistic models for some intelligence and attainment tests. Chicago: University of Chicago Press.

Rodrigues, M.W., Isotani, S. \& Zárate, L. E. (2018). Educational Data Mining: A review of evaluation process in the e-learning. Telematics and Informatics. 35(2018), 17011717. DOI: $10.1016 /$ j.tele.2018.04.015

Modelación matemática y algorítmica de los términos de la teoría del socioconstructivismo para un entorno educativo digital. Ivanov, I., Kosonogova, M., Cárdenas, J. 
Tsatsou, D., Vretos, N. \& Daras, P. (2019). Adaptive game-based learning in multi-agent educational settings. Journal of Computers in Education, 6(2), 215-239. DOI: 10.1007/s40692-018-0118-9

Tzuriel, D. (2000). The Seria-Think Instrument: Development of a Dynamic Test for Young Children. School Psychology International, 21 (2), 177-194.

Vázquez, P.G., \& Camacho, J.M. (2008). Teorías del aprendizaje y práctica docente. Sevilla: Wanceulen Educación.

Vygotsky, L. (2008). Pensamiento y Lenguaje. México: Quinto Sol.

Wilson, K. \& Nichols, Z. (2015). The Knewton Platform. A General-Purpose Adaptive Learning Infrastracture. A Knewton white paper.

Zuckerman, G. (2014). Developmental education. The Cambridge Handbook of CulturalHistorical Psychology (Cambridge Handbooks in Psychology), pp. 177-202. Cambridge: Cambridge University Press. doi:10.1017/CBO9781139028097.011

\section{Referencias de portales}

The Knewton Platform: <https://knewton.com>

Современная цифровая образовательная среда (El entorno educativo moderno digital) (en ruso) <http://neorusedu.ru/>

HotChalk, Inc. <https://www.hotchalk.com/>

Open Badges Movement <https://openbadges.org/>

Degreed: The Lifelong Learning Platform <https://degreed.com/>

SkilledUp: <https://skilledup.edu.au/>

Plataforma educativa Lynda.com <https://www.lynda.com/>

Plataforma educativa Udacity <https://www.udacity.com/>

Modelación matemática y algorítmica de los términos de la teoría del socioconstructivismo para un entorno educativo digital. Ivanov, I., Kosonogova, M., Cárdenas, J. 